TRANSACTIONS OF THE

AMERICAN MATHEMATICAL SOCIETY

Volume 356, Number 9, Pages 3455-3476

S 0002-9947(04)03500-7

Article electronically published on April 16, 2004

\title{
JACK POLYNOMIALS AND SOME IDENTITIES FOR PARTITIONS
}

\author{
MICHEL LASSALLE
}

\begin{abstract}
We prove an identity about partitions involving new combinatorial coefficients. The proof given is using a generating function. As an application we obtain the explicit expression of two shifted symmetric functions, related with Jack polynomials. These quantities are the moments of the " $\alpha$-content" random variable with respect to some transition probability distributions.
\end{abstract}

\section{INTRODUCTION}

A partition $\lambda=\left(\lambda_{1}, \ldots, \lambda_{n}\right)$ is a finite weakly decreasing sequence of positive integers, called parts. The number $n=l(\lambda)$ of parts is called the length of $\lambda$, and $|\lambda|=\sum_{i=1}^{n} \lambda_{i}$ the weight of $\lambda$. For any $i \geq 1, m_{i}(\lambda)=\operatorname{card}\left\{j: \lambda_{j}=i\right\}$ is the multiplicity of $i$ in $\lambda$. Clearly one has $l(\lambda)=\sum_{i} m_{i}(\lambda)$ and $|\lambda|=\sum_{i} i m_{i}(\lambda)$. We identify $\lambda$ with its Ferrers diagram $\left\{(i, j): 1 \leq i \leq l(\lambda), 1 \leq j \leq \lambda_{i}\right\}$ and set

$$
z_{\lambda}=\prod_{i \geq 1} i^{m_{i}(\lambda)} m_{i}(\lambda) !
$$

In this paper we consider the problem of evaluating sums of the following type:

$$
\sum_{|\mu|=n} \frac{1}{z_{\mu}} \prod_{k \geq 1}\left(S_{k}\right)^{m_{k}(\mu)}
$$

where $S_{k}$ is a formal series, depending on some indeterminates. The most elementary sum of this kind is well known [19]. Let $X$ be an indeterminate and $n$ a positive integer. We write

$$
(X)_{n}=X(X+1) \cdots(X+n-1), \quad[X]_{n}=X(X-1) \cdots(X-n+1)
$$

for the raising and lowering factorials and $\left(\begin{array}{l}X \\ n\end{array}\right)=[X]_{n} / n$ !. Choosing $S_{k}=X$, one has

$$
\sum_{|\mu|=n} \frac{X^{l(\mu)}}{z_{\mu}}=\left(\begin{array}{c}
X+n-1 \\
n
\end{array}\right)=\frac{(X)_{n}}{n !} .
$$

Received by the editors February 2, 2003.

2000 Mathematics Subject Classification. Primary 05A10, 05A17, 05E05, 33C52, 33C80.

Key words and phrases. Partitions, (shifted) symmetric functions, (shifted) Jack polynomials, transition probabilities. 
Let $X_{0}$ and $X=\left(X_{1}, X_{2}, \ldots\right)$ be (infinitely many) independent indeterminates. In [10] we considered another summation of the same type

$$
\sum_{|\mu|=n} \frac{1}{z_{\mu}} \prod_{k \geq 1}\left(\sum_{r \geq 0} u^{r} \frac{(k)_{r}}{r !} X_{r}\right)^{m_{k}(\mu)}
$$

and gave its formal series expansion.

In this paper we investigate a natural generalization of our previous result and give the formal series expansion of

$$
\sum_{|\mu|=n} \frac{1}{z_{\mu}} \prod_{k \geq 1}\left(\sum_{r, s \geq 0} u^{r} v^{s} \frac{(k)_{r}}{r !} \frac{(k)_{s}}{s !} X_{r+s}\right)^{m_{k}(\mu)} .
$$

The interest of this result is twofold. Firstly it involves new combinatorial objects whose background remains quite mysterious. Actually the given explicit expansion is written in terms of a new family of coefficients associated with partitions, which are themselves built in terms of another new family of positive integers. Both objects generalize classical binomial coefficients, but it should be emphasized that their combinatorial interpretation remains very obscure. It is an intriguing problem to clarify this underlying structure, which would at the same time provide a bijective proof of our result.

Secondly any specialization of the indeterminates $X_{i}$ may lead to a different application of our formula. Here our attention keeps mainly focused on symmetric function theory and (shifted) Jack polynomials.

Let $\alpha$ be some positive real number, $\lambda$ a partition and $A_{\lambda}$ the family of its so called " $\alpha$-contents" $\{j-1-(i-1) / \alpha,(i, j) \in \lambda\}$. Specializing $X_{i}$ to the $i$ th power sum symmetric function $p_{i}$ evaluated on $A_{\lambda}$, we obtain the series expansion of

$$
\frac{(x+y+1)_{\lambda}}{(x+y)_{\lambda}} \frac{(x)_{\lambda}}{(x+1)_{\lambda}},
$$

where $x, y$ are two indeterminates, and $(x)_{\lambda}$ is some natural generalization of the classical raising factorial.

It turns out that two families of shifted symmetric functions, closely related to (shifted) Jack polynomials, have a generating function of this type. Our formula provides an explicit expression for these shifted symmetric functions, thus proving (and improving) some earlier conjectures [14, 15]. These expressions are new even in the case $\alpha=1$, which corresponds to (shifted) Schur functions.

Our results have some connection with the works of Kerov [4, 5]. To any Young diagram are associated two discrete probability distributions, generalizing the Plancherel transition and co-transition classical probabilities. It turns out that the two shifted symmetric functions explicited here are the moments of the $\alpha$ content random variable with respect to these distributions.

Another consequence of our results is to allow a very easy computation of the expansion of Jack polynomials in terms of power sum symmetric functions. We hope to report about this application in a forthcoming paper.

\section{Combinatorial tools}

2.1. Positive integers. We first recall some results about a new family of positive integers, which we have introduced in [11]. 
Let $n, p, k$ be three integers with $1 \leq k \leq n$ and $0 \leq p \leq n$. We define

$$
\left(\begin{array}{l}
n \\
p
\end{array}\right)_{k}=\frac{n}{k} \sum_{r \geq 0}\left(\begin{array}{l}
p \\
r
\end{array}\right)\left(\begin{array}{c}
n-p \\
r
\end{array}\right)\left(\begin{array}{l}
n-r-1 \\
k-r-1
\end{array}\right) \text {. }
$$

We have obviously

$$
\left(\begin{array}{l}
n \\
p
\end{array}\right)_{k}=0 \quad \text { for } \quad k>n, \quad\left(\begin{array}{l}
n \\
p
\end{array}\right)_{1}=n, \quad\left(\begin{array}{l}
n \\
p
\end{array}\right)_{k}=\left(\begin{array}{c}
n \\
n-p
\end{array}\right)_{k} .
$$

These numbers generalize the classical binomial coefficients, since we have

$$
\left(\begin{array}{l}
n \\
0
\end{array}\right)_{k}=\left(\begin{array}{l}
n \\
k
\end{array}\right), \quad\left(\begin{array}{l}
n \\
1
\end{array}\right)_{k}=k\left(\begin{array}{l}
n \\
k
\end{array}\right), \quad\left(\begin{array}{l}
n \\
p
\end{array}\right)_{n}=\left(\begin{array}{l}
n \\
p
\end{array}\right),
$$

the last property being a direct consequence of the classical Chu-Vandermonde formula.

Other special values have been computed in [11], where it was also shown that the numbers $\left(\begin{array}{l}n \\ p\end{array}\right)_{k}$ are positive integers. A combinatorial interpretation of these numbers was given in [2].

In [11] we gave the following generating function:

$$
\sum_{k=1}^{n}\left(\begin{array}{l}
n \\
p
\end{array}\right)_{k}\left(\frac{z}{1-z}\right)^{k}=n z_{2} F_{1}\left[\begin{array}{c}
p+1, n-p+1 ; z \\
2
\end{array}\right]
$$

where as usual the classical Gauss hypergeometric function is denoted by

$$
{ }_{2} F_{1}\left[\begin{array}{c}
a, b \\
c
\end{array} ; z\right]=\sum_{i \geq 0} \frac{(a)_{i}(b)_{i}}{(c)_{i}} \frac{z^{i}}{i !}
$$

In other words, the generating function

$$
G_{n}(y, z)=\sum_{p=0}^{n} \sum_{k=1}^{n}\left(\begin{array}{l}
n \\
p
\end{array}\right) y_{k} y^{p}\left(\frac{z}{1-z}\right)^{k}
$$

can be written as

$$
G_{n}(y, z)=n z \sum_{p=0}^{n} y_{2}^{p} F_{1}\left[\begin{array}{c}
p+1, n-p+1 ; z \\
2
\end{array}\right] .
$$

It must be emphasized that $\left(\begin{array}{l}n \\ p\end{array}\right)_{k}$ is not defined for $p>n$, and that the generating function (2.1) is only valid for $0 \leq p \leq n$.

An explicit expression is known for $G_{n}(y, z)$ [27, 11], but we shall not need it. With $x=z /(1-z)$, i.e. $z=x /(1+x)$, we have

$$
\begin{aligned}
G_{n}(y, z)= & 2^{-n}\left((1+x)(1+y)+{\sqrt{(1+x)^{2}(1+y)^{2}-4 y(1+x)}}^{n}\right. \\
& +2^{-n}\left((1+x)(1+y)-{\sqrt{(1+x)^{2}(1+y)^{2}-4 y(1+x)}}^{n}-1-y^{n} .\right.
\end{aligned}
$$

2.2. New coefficients. The following notion has been introduced in previous papers [12, 13]. For $\lambda$ a partition and any integer $k \geq 1$, let $\left\langle\begin{array}{l}\lambda \\ k\end{array}\right\rangle$ denote the number of ways to choose $k$ different cells in the Ferrers diagram of $\lambda$, taking at least one cell from each row. It is easily seen that

$$
\left\langle\begin{array}{l}
\lambda \\
k
\end{array}\right\rangle=\sum_{\left(k_{i}\right)} \prod_{i=1}^{l(\lambda)}\left(\begin{array}{l}
\lambda_{i} \\
k_{i}
\end{array}\right)
$$


the sum being taken over all decompositions $k=\sum_{i=1}^{l(\lambda)} k_{i}$ with $k_{i} \neq 0$ for any $i$. The generating function for $\left\langle\begin{array}{l}\lambda \\ k\end{array}\right\rangle$ is the following:

$$
\begin{aligned}
\sum_{r \geq 1}\left\langle\begin{array}{l}
\lambda \\
r
\end{array}\right\rangle x^{r} & =\prod_{i=1}^{l(\lambda)}\left((1+x)^{\lambda_{i}}-1\right) \\
& =\prod_{i \geq 1}\left((1+x)^{i}-1\right)^{m_{i}(\lambda)} .
\end{aligned}
$$

Now in a strictly parallel way, for any integers $0 \leq p \leq|\lambda|$ and $k \geq 1$, we define

$$
\left\langle\begin{array}{l}
\lambda \\
p
\end{array}\right\rangle_{k}=\sum_{\left(p_{i}\right)} \sum_{\left(k_{i}\right)} \prod_{i=1}^{l(\lambda)}\left(\begin{array}{l}
\lambda_{i} \\
p_{i}
\end{array}\right)_{k_{i}}
$$

the sum being taken over all decompositions $p=\sum_{i=1}^{l(\lambda)} p_{i}, k=\sum_{i=1}^{l(\lambda)} k_{i}$ with $0 \leq$ $p_{i} \leq \lambda_{i}$ and $k_{i} \neq 0$ for any $i$. Observe that there is no such restriction for $p_{i}$.

This definition yields easily

$$
\left\langle\begin{array}{l}
\lambda \\
p
\end{array}\right\rangle_{k}=0 \quad \text { except if } \quad l(\lambda) \leq k \leq|\lambda| .
$$

Indeed it is obvious that $\left\langle\begin{array}{l}\lambda \\ p\end{array}\right\rangle_{k}=0$ for $k<l(\lambda)$, and since $\left(\begin{array}{l}n \\ p\end{array}\right)_{k}=0$ for $k>n$, we have also $\left\langle\begin{array}{l}\lambda \\ p\end{array}\right\rangle_{k}=0$ for $k>|\lambda|$. For instance $\left\langle\begin{array}{l}\lambda \\ p\end{array}\right\rangle_{1}=0$ except if $\lambda$ is a row partition $(n)$. In this case we have $\left\langle\begin{array}{c}(n) \\ p\end{array}\right\rangle_{k}=\left(\begin{array}{l}n \\ p\end{array}\right)_{k}$.

We have obviously

$$
\left\langle\begin{array}{l}
\lambda \\
0
\end{array}\right\rangle_{k}=\left\langle\begin{array}{l}
\lambda \\
k
\end{array}\right\rangle, \quad\left\langle\begin{array}{l}
\lambda \\
1
\end{array}\right\rangle_{k}=k\left\langle\begin{array}{l}
\lambda \\
k
\end{array}\right\rangle
$$

and also

$$
\left\langle\begin{array}{l}
\lambda \\
p
\end{array}\right\rangle_{k}=\left\langle\begin{array}{c}
\lambda \\
|\lambda|-p
\end{array}\right\rangle_{k}
$$

Finally it is not difficult to check that

$$
\left\langle\begin{array}{l}
\lambda \\
p
\end{array}\right\rangle_{|\lambda|}=\left(\begin{array}{c}
|\lambda| \\
p
\end{array}\right), \quad\left\langle\begin{array}{l}
\lambda \\
p
\end{array}\right\rangle_{|\lambda|-1}=\left(|\lambda|-m_{1}(\lambda)\right)\left[\left(\begin{array}{c}
|\lambda|-1 \\
p
\end{array}\right)+\left(\begin{array}{c}
|\lambda|-2 \\
p-2
\end{array}\right)\right] .
$$

For instance the first relation is a direct consequence of the Chu-Vandermonde formula. Indeed the definition easily implies

$$
\left\langle\begin{array}{l}
\lambda \\
p
\end{array}\right\rangle_{|\lambda|}=\sum_{\left(p_{i}\right)} \prod_{i=1}^{l(\lambda)}\left(\begin{array}{c}
\lambda_{i} \\
p_{i}
\end{array}\right)_{\lambda_{i}}=\sum_{\left(p_{i}\right)} \prod_{i=1}^{l(\lambda)}\left(\begin{array}{c}
\lambda_{i} \\
p_{i}
\end{array}\right) .
$$

As a straightforward consequence of their definition, the generating function for the integers $\left\langle\begin{array}{l}\lambda \\ p\end{array}\right\rangle_{k}$ is the following:

$$
\sum_{p=0}^{|\lambda|} \sum_{k=l(\lambda)}^{|\lambda|}\left\langle\begin{array}{l}
\lambda \\
p
\end{array}\right\rangle_{k} y^{p}\left(\frac{z}{1-z}\right)^{k}=\prod_{i=1}^{l(\lambda)} G_{\lambda_{i}}(y, z)=\prod_{i \geq 1}\left(G_{i}(y, z)\right)^{m_{i}(\lambda)} .
$$

One has easily $G_{i}\left(0, \frac{x}{1+x}\right)=(1+x)^{i}-1$. Thus for $y=0$ we recover the generating function of $\left\langle\begin{array}{l}\lambda \\ 0\end{array}\right\rangle_{k}=\left\langle\begin{array}{l}\lambda \\ k\end{array}\right\rangle$. 
Observe that $\left\langle\begin{array}{l}\lambda \\ p\end{array}\right\rangle_{k}$ is not defined for $p>|\lambda|$. An interesting problem is to get a combinatorial interpretation of the positive integers $\left\langle{ }_{p}^{\lambda}\right\rangle_{k}$.

2.3. Auxiliary polynomials. Let $X=\left(X_{1}, X_{2}, \ldots\right)$ be (infinitely many) independent indeterminates. In [10, 11, 12] for any integers $n, k \geq 1$ we have defined

$$
P_{n k}(X)=\sum_{|\mu|=n} \frac{\left\langle\begin{array}{l}
\mu \\
k
\end{array}\right\rangle}{z_{\mu}} \prod_{i \geq 1} X_{i}^{m_{i}(\mu)} .
$$

For $k=0$ we got $P_{n 0}(X)=0(n \neq 0)$, and $P_{00}(X)=1$.

In a strictly parallel way, for any integers $n \geq 1, k \geq 1$ and $0 \leq p \leq n$ we set

$$
P_{n p k}(X)=\sum_{|\mu|=n} \frac{\left\langle\begin{array}{l}
\mu \\
p
\end{array}\right\rangle_{k}}{z_{\mu}} \prod_{i \geq 1} X_{i}^{m_{i}(\mu)} .
$$

Since $\left\langle{ }_{p}^{\mu}\right\rangle_{k}=0$ for $k<l(\mu)$, this sum is restricted to partitions such that $l(\mu) \leq k$. Hence $P_{n p k}(X)$ is a polynomial in $X$ with degree $k$. Similarly since $\left\langle{ }_{p}^{\mu}\right\rangle_{k}=0$ for $k>|\mu|$, one has $P_{n p k}(X)=0$ for $k>n$.

For $k=0$ it is natural to extend the previous definition by the convention $P_{n p 0}(X)=0$ with the only exception $P_{000}(X)=1$. Note that $P_{n p k}(X)$ is not defined for $p>n$.

For $k=1$ we have $P_{n p 1}(X)=X_{n}$ and for $k=n$,

$$
P_{n p n}(X)=\left(\begin{array}{l}
n \\
p
\end{array}\right) \sum_{|\mu|=n} \frac{1}{z_{\mu}} \prod_{i \geq 1} X_{i}^{m_{i}(\mu)}=\left(\begin{array}{l}
n \\
p
\end{array}\right) P_{n n}(X) .
$$

Finally we have $P_{n 0 k}(X)=P_{n k}(X)$ and $P_{n 1 k}(X)=k P_{n k}(X)$. It is also obvious that $P_{n p k}(X)=P_{n, n-p, k}(X)$.

\section{MAin identity}

Let $X_{0}$ and $X=\left(X_{1}, X_{2}, \ldots\right)$ be (infinitely many) independent indeterminates. We give the explicit evaluation of

$$
\sum_{|\mu|=n} \frac{1}{z_{\mu}} \prod_{k \geq 1}\left(S_{k}\right)^{m_{k}(\mu)}
$$

when the formal series $S_{k}$ is chosen to be

$$
S_{k}=\sum_{r, s \geq 0} u^{r} v^{s} \frac{(k)_{r}}{r !} \frac{(k)_{s}}{s !} X_{r+s} .
$$

Theorem 3.1. For any integer $n \geq 1$ we have

$$
\begin{aligned}
\sum_{|\mu|=n} \frac{1}{z_{\mu}} \prod_{k \geq 1}\left(\sum_{r, s \geq 0} u^{r} v^{s} \frac{(k)_{r}}{r !} \frac{(k)_{s}}{s !} X_{r+s}\right)^{m_{k}(\mu)} & \\
& =\sum_{p, q \geq 0} u^{p} v^{q}\left(\sum_{k=0}^{\min (n, p+q)}\left(\begin{array}{c}
X_{0}+n-1 \\
n-k
\end{array}\right) P_{p+q, p, k}(X)\right) .
\end{aligned}
$$


Proof. Denote by $L(n)$ (resp. $R(n)$ ) the left (resp. right)-hand side of this identity. On the right-hand side, with $b=a /(1-a)$ and $w=u / v$, we get

$$
\begin{aligned}
\sum_{n \geq 1} a^{n} R(n) & =\sum_{p, q \geq 0} u^{p} v^{q} \sum_{k=0}^{p+q}\left(\sum_{n \geq k} a^{n}\left(\begin{array}{c}
X_{0}+n-1 \\
n-k
\end{array}\right)\right) P_{p+q, p, k}(X) \\
& =(1-a)^{-X_{0}} \sum_{p, q \geq 0} u^{p} v^{q} \sum_{k=0}^{p+q} b^{k}\left(\sum_{|\mu|=p+q} \frac{\left\langle\begin{array}{l}
\mu \\
p
\end{array}\right\rangle_{k}}{z_{\mu}} \prod_{i \geq 1} X_{i}{ }^{m_{i}(\mu)}\right) \\
& =(1-a)^{-X_{0}} \sum_{\mu} v^{|\mu|} \sum_{p=0}^{|\mu|} \sum_{k=0}^{|\mu|} w^{p} b^{k} \frac{\left\langle\begin{array}{l}
\mu \\
p
\end{array}\right\rangle_{k}}{z_{\mu}} \prod_{i \geq 1} X_{i}^{m_{i}(\mu)} .
\end{aligned}
$$

Using the generating function (2.3) for $\left\langle{ }_{p}^{\mu}\right\rangle_{k}$, we obtain

$$
\begin{aligned}
\sum_{n \geq 1} a^{n} R(n) & =(1-a)^{-X_{0}} \sum_{m_{1}, m_{2}, \ldots \geq 0} \prod_{k \geq 1} \frac{1}{m_{k} !}\left(v^{k} \frac{X_{k}}{k} G_{k}(w, a)\right)^{m_{k}} \\
& =(1-a)^{-X_{0}} \prod_{k \geq 1} \exp \left(v^{k} \frac{X_{k}}{k} G_{k}(w, a)\right) \\
& =(1-a)^{-X_{0}} \exp \left(\sum_{k \geq 1} v^{k} \frac{X_{k}}{k} G_{k}(w, a)\right) .
\end{aligned}
$$

On the left-hand side of the identity we have

$$
\begin{aligned}
\sum_{n \geq 1} a^{n} L(n) & =\sum_{\mu} \frac{a^{|\mu|}}{z_{\mu}} \prod_{k \geq 1}\left(X_{0}+\sum_{t \geq 1} X_{t} \sum_{r=0}^{t} u^{r} v^{t-r} \frac{(k)_{r}}{r !} \frac{(k)_{t-r}}{(t-r) !}\right)^{m_{k}(\mu)} \\
& =\sum_{m_{1}, m_{2}, \ldots \geq 0} \prod_{k \geq 1} \frac{1}{m_{k} !}\left[\frac{a^{k}}{k}\left(X_{0}+\sum_{t \geq 1} v^{t} X_{t} \sum_{r=0}^{t} w^{r} \frac{(k)_{r}}{r !} \frac{(k)_{t-r}}{(t-r) !}\right)\right]^{m_{k}} \\
& =(1-a)^{-X_{0}} \prod_{k \geq 1} \exp \left(\frac{a^{k}}{k} \sum_{t \geq 1} v^{t} X_{t} \sum_{r=0}^{t} w^{r} \frac{(k)_{r}}{r !} \frac{(k)_{t-r}}{(t-r) !}\right) \\
& =(1-a)^{-X_{0}} \exp \left[\sum_{t \geq 1} v^{t} X_{t} \sum_{r=0}^{t} w^{r}\left(\sum_{k \geq 1} \frac{a^{k}}{k} \frac{(k)_{r}}{r !} \frac{(k)_{t-r}}{(t-r) !}\right)\right]
\end{aligned}
$$

Thus it is enough to prove

$$
G_{n}(w, a)=n \sum_{r=0}^{n} w^{r}\left(\sum_{i \geq 1} \frac{a^{i}}{i} \frac{(i)_{r}}{r !} \frac{(i)_{n-r}}{(n-r) !}\right) .
$$

But obviously

$$
\sum_{i \geq 1} \frac{a^{i}}{i} \frac{(i)_{r}}{r !} \frac{(i)_{n-r}}{(n-r) !}=a_{2} F_{1}\left[\begin{array}{c}
r+1, n-r+1 \\
2
\end{array} ; a .\right.
$$

We conclude by applying relation (2.2). 
Theorem 3.1 immediately yields

$$
\begin{aligned}
\sum_{|\mu|=n}(-1)^{n-l(\mu)} \frac{1}{z_{\mu}} \prod_{k \geq 1} & \left(\sum_{r, s \geq 0} u^{r} v^{s} \frac{(k)_{r}}{r !} \frac{(k)_{s}}{s !} X_{r+s}\right)^{m_{k}(\mu)} \\
& =\sum_{p, q \geq 0} u^{p} v^{q}\left(\sum_{k=0}^{\min (n, p+q)}(-1)^{k}\left(\begin{array}{c}
X_{0}-k \\
n-k
\end{array}\right) P_{p+q, p, k}(-X)\right) .
\end{aligned}
$$

It is an open question whether the right-hand side may be expressed in terms of the quantities $P_{p+q, p, k}(X)$. This is the case for $v=0$ ([10], Theorem 2, p. 301). Then one has

$$
\begin{aligned}
\sum_{|\mu|=n}(-1)^{n-l(\mu)} \frac{1}{z_{\mu}} \prod_{k \geq 1}\left(\sum_{r \geq 0} u^{r} \frac{(k)_{r}}{r !} X_{r}\right)^{m_{k}(\mu)} & \\
& =\sum_{p \geq 0} u^{p}\left(\sum_{k=0}^{\min (n, p)}\left(\begin{array}{c}
X_{0}-p \\
n-k
\end{array}\right) P_{p k}(X)\right) .
\end{aligned}
$$

This is a consequence of the following nontrivial property ([1], Corollaire 5):

$$
\sum_{k=l(\mu)}^{\min (n,|\mu|)}\left(\begin{array}{c}
X_{0}-|\mu| \\
n-k
\end{array}\right)\left\langle\begin{array}{l}
\mu \\
k
\end{array}\right)=\sum_{k=l(\mu)}^{\min (n,|\mu|)}(-1)^{k-l(\mu)}\left(\begin{array}{c}
X_{0}-k \\
n-k
\end{array}\right)\left\langle\begin{array}{l}
\mu \\
k
\end{array}\right) .
$$

It would be interesting to obtain a generalization of this identity for $\left\langle\begin{array}{l}\mu \\ p\end{array}\right\rangle_{k}$.

\section{First specialization}

Various applications of Theorem 3.1 may be obtained by using different specializations of the indeterminates $X_{i}$. In this section we shall consider the simplest case, obtained when all the $X_{i}, i \geq 0$, are equal.

Let $x$ be an indeterminate. In [13] (Theorem 1, p. 461) we gave a bijective proof (due to Rodica Simion) of the identity

$$
\sum_{|\mu|=n}\left\langle\begin{array}{l}
\mu \\
k
\end{array}\right\rangle \frac{x^{l(\mu)}}{z_{\mu}}=\left(\begin{array}{c}
n-1 \\
k-1
\end{array}\right)\left(\begin{array}{c}
x+k-1 \\
k
\end{array}\right) .
$$

Since the Stirling numbers of the first kind $s(k, r)$ are defined by the generating function

$$
(x)_{k}=\sum_{r \geq 1}|s(k, r)| x^{r}
$$

this identity is equivalent to

$$
\left(\begin{array}{c}
n-1 \\
k-1
\end{array}\right)|s(k, r)|=k ! \sum_{\substack{|\mu|=n \\
l(\mu)=r}} \frac{\left\langle\begin{array}{l}
\mu \\
k
\end{array}\right\rangle}{z_{\mu}} .
$$

We generalize this result as follows (the previous case may be recovered by choosing $p=0)$. 
Theorem 4.1. Let $x$ be an indeterminate. For any nonnegative integers $n, p, r$, we have

$$
\sum_{|\mu|=n}\left\langle\begin{array}{l}
\mu \\
p
\end{array}\right\rangle_{k} \frac{x^{l(\mu)}}{z_{\mu}}=\frac{k}{n}\left(\begin{array}{l}
n \\
p
\end{array}\right)_{k}\left(\begin{array}{c}
x+k-1 \\
k
\end{array}\right) .
$$

Proof. With the specialization $X_{i}=x$ for any $i \geq 0$, the left-hand side of the identity of Theorem 3.1 reads

$$
\begin{aligned}
\sum_{|\mu|=n} & \frac{1}{z_{\mu}} \prod_{k \geq 1}\left(\sum_{r, s \geq 0} u^{r} v^{s} \frac{(k)_{r}}{r !} \frac{(k)_{s}}{s !} x\right)^{m_{k}(\mu)} \\
& =\sum_{|\mu|=n} \frac{x^{l(\mu)}}{z_{\mu}} \prod_{k \geq 1}\left((1-u)^{-k}(1-v)^{-k}\right)^{m_{k}(\mu)} \\
& =\left(\begin{array}{c}
x+n-1 \\
n
\end{array}\right)(1-u)^{-n}(1-v)^{-n} \\
& =\left(\begin{array}{c}
x+n-1 \\
n
\end{array}\right)\left(\sum_{p, q \geq 0} u^{p} v^{q} \frac{(n)_{p}}{p !} \frac{(n)_{q}}{q !}\right) .
\end{aligned}
$$

By identification of the coefficients in $u$ and $v$, we immediately obtain for $p, q>0$,

$$
\begin{aligned}
\left(\begin{array}{c}
x+n-1 \\
n
\end{array}\right) \frac{(n)_{p}}{p !} \frac{(n)_{q}}{q !} & =\sum_{k=1}^{\min (n, p+q)}\left(\begin{array}{c}
x+n-1 \\
n-k
\end{array}\right) P_{p+q, p, k}(x, \ldots, x) \\
& =\sum_{k=1}^{\min (n, p+q)}\left(\begin{array}{c}
x+n-1 \\
n-k
\end{array}\right)\left(\sum_{|\mu|=p+q}\left\langle\begin{array}{c}
\mu \\
p
\end{array}\right\rangle_{k} \frac{x^{l(\mu)}}{z_{\mu}}\right) .
\end{aligned}
$$

On the other hand, it is easily proved that we have

$$
\left(\begin{array}{c}
x+n-1 \\
n
\end{array}\right) \frac{(n)_{p}}{p !} \frac{(n)_{q}}{q !}=\sum_{k=1}^{\min (n, p+q)}\left(\begin{array}{c}
x+n-1 \\
n-k
\end{array}\right) \frac{k}{p+q}\left(\begin{array}{c}
p+q \\
p
\end{array}\right)_{k}\left(\begin{array}{c}
x+k-1 \\
k
\end{array}\right)
$$

or equivalently

$$
\frac{(n)_{p}}{p !} \frac{(n)_{q}}{q !}=\sum_{k=1}^{\min (n, p+q)}\left(\begin{array}{l}
n \\
k
\end{array}\right) \frac{k}{p+q}\left(\begin{array}{c}
p+q \\
p
\end{array}\right)_{k} .
$$

Indeed for $p=0$ this is the classical Chu-Vandermonde formula. The property is then a direct consequence of the following recurrence relation ([11, Lemma 3.2):

$$
p\left(\begin{array}{c}
p+q \\
p
\end{array}\right)_{k}=(q+1)\left(\begin{array}{c}
p+q \\
p-1
\end{array}\right)_{k}-\frac{p+q}{p+q-1}(q-p+1)\left(\begin{array}{c}
p+q-1 \\
p-1
\end{array}\right)_{k} .
$$

But now if for a sequence of functions $f_{k}^{(p q)}(x), 1 \leq k \leq p+q$, the relation

$$
\sum_{k=1}^{\min (n, p+q)}\left(\begin{array}{c}
x+n-1 \\
n-k
\end{array}\right) f_{k}^{(p q)}(x)=0
$$

is satisfied for any $n \geq 1$, by choosing successively $n=1,2, \ldots, p+q$ one has iteratively $f_{k}^{(p q)}=0$, and we can conclude. 
Theorem 4.1 can be equivalently stated as

$$
\frac{k}{n}\left(\begin{array}{l}
n \\
p
\end{array}\right)_{k}|s(k, r)|=k ! \sum_{\substack{|\mu|=n \\
l(\mu)=r}} \frac{\left\langle\begin{array}{l}
\mu \\
p
\end{array}\right\rangle_{k}}{z_{\mu}}
$$

It would be interesting to obtain a bijective proof, in the same vein than Simion's.

\section{MAIN SPECIALIZATION}

We now consider another specialization of Theorem 3.1 in the framework of (shifted) Jack polynomials.

Let $\alpha$ be some positive real number, and $\lambda$ an arbitrary partition. For any cell $(i, j)$ of $\lambda$ we define the " $\alpha$-content"

$$
c_{i j}^{\alpha}=j-1-(i-1) / \alpha .
$$

We consider the following natural generalization of the raising and lowering factorial:

$$
(x)_{\lambda}=\prod_{(i, j) \in \lambda}\left(x+c_{i j}^{\alpha}\right), \quad[x]_{\lambda}=\prod_{(i, j) \in \lambda}\left(x-c_{i j}^{\alpha}\right) .
$$

For any integer $k \geq 0$ we define

$$
d_{k}(\lambda)=\sum_{(i, j) \in \lambda}\left(c_{i j}^{\alpha}\right)^{k}
$$

For any integers $n, p, k \geq 0$ we set

$$
F_{n p k}(\lambda)=P_{n p k}\left(d_{1}(\lambda), d_{2}(\lambda), d_{3}(\lambda), \ldots\right)=\sum_{|\mu|=n} \frac{\left\langle\begin{array}{l}
\mu \\
p
\end{array}\right\rangle_{k}}{z_{\mu}} d_{\mu}(\lambda)
$$

and $F_{n k}(\lambda)=F_{n 0 k}(\lambda)$, with the obvious notation

$$
d_{\mu}(\lambda)=\prod_{i \geq 1} d_{i}(\lambda)^{m_{i}(\mu)}
$$

In other words we choose the following specialization:

$$
X_{0}=d_{0}(\lambda)=|\lambda|, \quad X_{k}=d_{k}(\lambda) \quad(k \geq 1),
$$

though the quantities $d_{k}(\lambda)$ are no longer independent indeterminates.

In [10] we have shown the following development:

$$
\frac{(x+y)_{\lambda}}{(y)_{\lambda}}=\sum_{i, j \geq 0}(-1)^{j} \frac{x^{i}}{y^{i+j}}\left(\sum_{k=0}^{\min (i, j)}\left(\begin{array}{c}
|\lambda|-j \\
i-k
\end{array}\right) F_{j k}(\lambda)\right),
$$

where the sum takes over any $j \geq 0$ and not only over $|\lambda|-j \geq 0$.

An analogous result can be deduced from Theorem 3.1.

Theorem 5.1. Let $x, y$ be two indeterminates. Then we have

$$
\begin{aligned}
& \frac{(x+y+1)_{\lambda}}{(x+y)_{\lambda}} \frac{(x)_{\lambda}}{(x+1)_{\lambda}}=\sum_{n \geq 0}\left(-\frac{y}{x^{2}}\right)^{n}\left(1+\frac{y+1}{x}\right)^{-n} \\
& \quad \times \sum_{p, q \geq 0}\left(\frac{-1}{x}\right)^{p+q}\left(1+\frac{y+1}{x}\right)^{-q}\left(\sum_{k=0}^{\min (n, p+q)}\left(\begin{array}{c}
|\lambda|+n-1 \\
n-k
\end{array}\right) F_{p+q, p, k}(\lambda)\right) .
\end{aligned}
$$


Proof. With $c_{i j}=c_{i j}^{\alpha}$ for short, the left-hand side can be written as

$$
\begin{aligned}
\text { LHS } & =\prod_{(i, j) \in \lambda} \frac{\left(x+y+1+c_{i j}\right)}{\left(x+y+c_{i j}\right)} \frac{\left(x+c_{i j}\right)}{\left(x+1+c_{i j}\right)} \\
& =\prod_{(i, j) \in \lambda}\left(1+\frac{y}{\left(x+y+1+c_{i j}\right)\left(x+c_{i j}\right)}\right)^{-1} .
\end{aligned}
$$

Setting $u=-1 / x, v=-1 /(x+y+1)$, and using the classical series expansion

$$
\log (1-a)=-\sum_{k \geq 1} \frac{a^{k}}{k}
$$

we get

$$
\begin{aligned}
\text { LHS } & =\prod_{(i, j) \in \lambda} \exp \left[\sum_{k \geq 1} \frac{(-y)^{k}}{k}\left(\left(x+y+1+c_{i j}\right)\left(x+c_{i j}\right)\right)^{-k}\right] \\
& =\exp \left[\sum_{(i, j) \in \lambda}\left(\sum_{k \geq 1} \frac{(-y u v)^{k}}{k}\left(1-c_{i j} u\right)^{-k}\left(1-c_{i j} v\right)^{-k}\right)\right] .
\end{aligned}
$$

Then using the series expansion

$$
(1-a)^{-k}=\sum_{r \geq 0} a^{r} \frac{(k)_{r}}{r !}
$$

we obtain

$$
\begin{aligned}
\text { LHS } & =\exp \left[\sum_{k \geq 1} \frac{(-y u v)^{k}}{k}\left(\sum_{r, s \geq 0} u^{r} v^{s} \frac{(k)_{r}}{r !} \frac{(k)_{s}}{s !} d_{r+s}(\lambda)\right)\right] \\
& =\prod_{k \geq 1}\left(\sum_{m_{k} \geq 0} \frac{1}{m_{k} !}\left[\frac{(-y u v)^{k}}{k}\left(\sum_{r, s \geq 0} u^{r} v^{s} \frac{(k)_{r}}{r !} \frac{(k)_{s}}{s !} d_{r+s}(\lambda)\right)\right]^{m_{k}}\right) \\
& =\sum_{\mu} \frac{(-y u v)^{|\mu|}}{z_{\mu}} \prod_{k \geq 1}\left(\sum_{r, s \geq 0} u^{r} v^{s} \frac{(k)_{r}}{r !} \frac{(k)_{s}}{s !} d_{r+s}(\lambda)\right)^{m_{k}(\mu)} .
\end{aligned}
$$

We conclude by applying Theorem 3.1 with the specialization $X_{k}=d_{k}(\lambda)$, which gives

$$
\text { LHS }=\sum_{n \geq 0}(-y u v)^{n} \sum_{p, q \geq 0} u^{p} v^{q}\left(\sum_{k=0}^{\min (n, p+q)}\left(\begin{array}{c}
|\lambda|+n-1 \\
n-k
\end{array}\right) F_{p+q, p, k}(\lambda)\right) .
$$

Corollary 5.2. Let $x, y$ be two indeterminates. Then we have

$$
\frac{(x+y+1)_{\lambda}}{(x+y)_{\lambda}} \frac{(x)_{\lambda}}{(x+1)_{\lambda}}=\sum_{r \geq 0} c_{r}\left(\frac{-1}{x}\right)^{r}
$$


with

$$
\begin{aligned}
c_{r}=\sum_{\substack{n, p, q \geq 0 \\
2 n+p+q \leq r}}(-y)^{n}(y+1)^{p} & \left(\begin{array}{c}
n+p+q-1 \\
p
\end{array}\right) \\
& \left.\times\left(\begin{array}{c}
\min (n, r-2 n-p) \\
\sum_{k=0}^{|\lambda|+n-1} \\
n-k
\end{array}\right) F_{r-2 n-p, q, k}(\lambda)\right) .
\end{aligned}
$$

Remark 5.3. Observe the situation for low indices. One has $c_{0}=1$ since $F_{000}(\lambda)=$ 1 , and $c_{1}=0$ since $F_{1 q 0}(\lambda)=0$.

This result is useful in the study of Jack polynomials, allowing to prove some conjectures stated in [14, 15]. The proofs will be given in Sections 8 and 9 .

\section{Symmetric FunCtions}

Let $A=\left\{a_{1}, a_{2}, a_{3}, \ldots\right\}$ a (possibly infinite) set of independent indeterminates ( $A$ is called an alphabet). The generating functions

$$
\begin{aligned}
& E_{t}(A)=\prod_{a \in A}(1+t a)=\sum_{k \geq 0} t^{k} e_{k}(A), \\
& H_{t}(A)=\prod_{a \in A} \frac{1}{1-t a}=\sum_{k \geq 0} t^{k} h_{k}(A), \\
& P_{t}(A)=\sum_{a \in A} \frac{a}{1-t a}=\sum_{k \geq 1} t^{k-1} p_{k}(A)
\end{aligned}
$$

define symmetric functions known as elementary, complete and power sums, respectively. For any partition $\mu$, we define functions $e_{\mu}, h_{\mu}$ or $p_{\mu}$ by

$$
f_{\mu}=\prod_{i=1}^{l(\mu)} f_{\mu_{i}}=\prod_{k \geq 1} f_{k}^{m_{k}(\mu)}
$$

where $f_{i}$ stands for $e_{i}, h_{i}$ or $p_{i}$. The monomial symmetric function $m_{\mu}(A)$ is defined as the sum of all distincts monomials $\prod_{i} a_{i}^{m_{i}}$ such that $\left(m_{i}\right)$ is a permutation of $\mu[19]$.

When $A$ is infinite, each of the three sets of functions $e_{i}(A), h_{i}(A)$ or $p_{i}(A)$ forms an algebraic basis of $\mathbf{S}[A]$, the symmetric algebra of $A$. Each of the sets of functions $e_{\mu}(A), h_{\mu}(A), p_{\mu}(A)$ is a linear basis of this algebra. It is thus possible to define the symmetric algebra $\mathbf{S}$ abstractly, as the $\mathbf{R}$-algebra generated by the functions $e_{i}, h_{i}$ or $p_{i}$

This is no longer true when $A$ is finite, a situation which is often encountered. In that case the functions $e_{\mu}(A)$ (resp. $h_{\mu}(A), p_{\mu}(A)$ ) are no longer linearly independent. We have encountered such a situation with the previous specialization $X_{k}=d_{k}(\lambda)$. Let

$$
A_{\lambda}=\left\{c_{i j}^{\alpha}=j-1-(i-1) / \alpha,(i, j) \in \lambda\right\}
$$

denote the finite alphabet of $\alpha$-contents of $\lambda$. By definition we have

$$
d_{k}(\lambda)=p_{k}\left(A_{\lambda}\right) \quad(k \geq 1), \quad d_{\mu}(\lambda)=p_{\mu}\left(A_{\lambda}\right) .
$$

Thus the right-hand sides of the relation of Theorem 5.1 and of relation (5.1) are symmetric functions of the alphabet $A_{\lambda}$. The expressions are given in terms of 
the power sums $p_{\mu}\left(A_{\lambda}\right)$, but it might be also interesting to convert them in terms of any other symmetric functions of $A_{\lambda}$.

With this conversion in mind, we are led to the following two open problems. Let $A=\left\{a_{1}, a_{2}, a_{3}, \ldots\right\}$ be any (finite or infinite) alphabet. Choose the specialization $X_{k}=p_{k}(A)(k \geq 1)$. By definition for any $n, k \geq 0$ we have

$$
P_{n k}(-X)=\sum_{|\mu|=n}(-1)^{l(\mu)} \frac{\left\langle\begin{array}{l}
\mu \\
k
\end{array}\right\rangle}{z_{\mu}} p_{\mu}(A) .
$$

In [10] (Lemma 2, p. 306) we have shown that

$$
P_{n k}(-X)=(-1)^{k} \sum_{\substack{|\mu|=n \\ l(\mu)=k}} m_{\mu}(A) .
$$

Let $\omega$ be the involution defined by $\omega\left(p_{r}\right)=(-1)^{r-1} p_{r}$. One has $\omega\left(P_{n k}(X)\right)=$ $(-1)^{n} P_{n k}(-X)$, hence

$$
P_{n k}(X)=(-1)^{n-k} \sum_{\substack{|\mu|=n \\ l(\mu)=k}} f_{\mu}(A),
$$

with $f_{\mu}$ the fourth classical basis of "forgotten" symmetric functions ([19], p. 22).

Problem 1. Evaluate $P_{n p k}(-X)$ in terms of monomial symmetric functions of $A$.

Using ACE [26] there is some experimental evidence that

$$
P_{n p k}(-X)=(-1)^{k} \sum_{\substack{|\mu|=n \\ l(\mu)=k}} \chi_{\mu} m_{\mu}(A),
$$

with $\chi_{\mu}$ some polynomial in the multiplicities of $\mu$. It seems that for $p \leq 3$ one has

$$
\chi_{\mu}=\left(\begin{array}{c}
k+p-1 \\
p
\end{array}\right)-\left(\begin{array}{c}
k+p-3 \\
p-2
\end{array}\right) m_{1}(\mu)-\left(\begin{array}{c}
k+p-4 \\
p-3
\end{array}\right) m_{2}(\mu) .
$$

Now if we define the alphabet

$$
B_{0}=\left\{a_{1} /\left(1-a_{1}\right), a_{2} /\left(1-a_{2}\right), a_{3} /\left(1-a_{3}\right), \ldots\right\},
$$

it was proved in [1] (Theorem 2) that for any $k \geq 1$, one has

$$
\begin{gathered}
p_{k}\left(B_{0}\right)=\sum_{n \geq k}\left(\begin{array}{l}
n-1 \\
k-1
\end{array}\right) p_{n}(A), \\
h_{k}\left(B_{0}\right)=\sum_{n \geq k} P_{n k}(X), \quad e_{k}\left(B_{0}\right)=(-1)^{k} \sum_{n \geq k} P_{n k}(-X) .
\end{gathered}
$$

Problem 2. For any integer $p \geq 0$, find an alphabet $B_{p}$ such that for any $k \geq 1$,

$$
p_{k}\left(B_{p}\right)=\sum_{n \geq \max (k, p)} \frac{k}{n}\left(\begin{array}{l}
n \\
p
\end{array}\right) p_{k}(A) .
$$

Give the expansion of $h_{k}\left(B_{p}\right)$ (resp. $e_{k}\left(B_{p}\right)$ ) in terms of the quantities $P_{n p k}(X)$ (resp. $\left.P_{n p k}(-X)\right)$. 
For any two alphabets $A$ and $B$, their difference $A-B$ (which is not their difference as sets) is defined by

$$
E_{t}(A-B)=E_{t}(A) E_{t}(B)^{-1}, \quad H_{t}(A-B)=H_{t}(A) H_{t}(B)^{-1} .
$$

In Sections 8 and 9 we shall need the following result about Lagrange interpolation [9].

Lagrange Lemma. Let $A$ and $B$ be two finite alphabets with respective cardinals $n$ and $m$. For any integer $r \geq 0$ we have

$$
\sum_{a \in A} a^{r} \frac{\prod_{b \in B}(a-b)}{\prod_{c \in A, c \neq a}(a-c)}=h_{m-n+r+1}(A-B) .
$$

Alain Lascoux 9 mentions that when $B$ is empty, this result was already known to Euler.

\section{SHIFTED SYMmetric FUnCTIONS}

Though the theory of symmetric functions goes back to the early 19th century, the notion of "shifted symmetric" functions is very recent. We refer to [7, 8, 24, $21,22,23$ and to references given there. We shall follow the presentation given in 21, 22, 23].

Let $\alpha$ be some fixed positive real number and $\mathbf{F}$ the field of rational functions in $\alpha$. A polynomial in $n$ variables $\left(x_{1}, x_{2}, \ldots, x_{n}\right)$ with coefficients in $\mathbf{F}$ is said to be shifted symmetric if it is symmetric in the "shifted variables" $x_{i}-i / \alpha$. Let $\mathbf{S}_{n}^{*}$ denote this subalgebra of $\mathbf{F}\left[x_{1}, x_{2}, \ldots, x_{n}\right]$.

Now let $X=\left\{x_{1}, x_{2}, x_{3}, \ldots\right\}$ be an infinite alphabet. Consider the morphism from $\mathbf{S}_{n+1}^{*}$ to $\mathbf{S}_{n}^{*}$ given by $x_{n+1}=0$. In analogy with symmetric functions, the shifted symmetric algebra $\mathbf{S}^{*}[X]$ is defined as the projective limit of the algebras $\mathbf{S}_{n}^{*}$ with respect to this morphism. In other words a "shifted symmetric function" $f \in \mathbf{S}^{*}[X]$ is a family $\left\{f_{n}, n \geq 1\right\}$ with the following two properties:

(i) $f_{n} \in \mathbf{S}_{n}^{*}$ (shifted symmetry),

(ii) $f_{n+1}\left(x_{1}, x_{2}, \ldots, x_{n}, 0\right)=f_{n}\left(x_{1}, x_{2}, \ldots, x_{n}\right)$ (stability).

For instance if for any integer $k \geq 1$ we define the "shifted power sums" by

$$
p_{k}^{*}(x)=\sum_{i \geq 1}\left(\left[x_{i}-(i-1) / \alpha\right]_{k}-[-(i-1) / \alpha]_{k}\right),
$$

these polynomials algebraically generate $\mathbf{S}^{*}[X]$.

Any element $f \in \mathbf{S}^{*}[X]$ may be evaluated at any sequence $x=\left(x_{1}, x_{2}, \ldots\right)$ with finitely many nonzero terms, hence at any partition $\lambda$. Moreover by analyticity, $f$ is entirely determined by its restriction $f(\lambda)$ to partitions. From now on we shall perform this identification, and consider $\mathbf{S}^{*}[X]$ as a function algebra $\mathbf{S}^{*}[\mathcal{P}]$ on the set $\mathcal{P}$ of partitions. In this approach, a partition is no longer a parameter but an argument. The following lemma is crucial ([14], p. 151). The argument is taken from [6] (Lemma 7.1).

Lemma 7.1. For any integer $k \geq 1$, the function $\lambda \rightarrow d_{k}(\lambda)$ defines a shifted symmetric function. 
Proof. Let $t(k, m)$ denote the inverse matrix of $s(k, m)$, the matrix of Stirling numbers of the first kind, that is, $x^{k}=\sum_{m=1}^{k} t(k, m)[x]_{m}$. We have

$$
d_{k}=\sum_{m=1}^{k} t(k, m) \frac{p_{m+1}^{*}}{m+1} .
$$

Indeed we can write

$$
\begin{aligned}
d_{k}(\lambda) & =\sum_{m=1}^{k} \sum_{(i, j) \in \lambda} t(k, m)[j-1-(i-1) / \alpha]_{m} \\
& =\sum_{m=1}^{k} \frac{t(k, m)}{m+1} \sum_{i=1}^{l(\lambda)}\left(\left[\lambda_{i}-(i-1) / \alpha\right]_{m+1}-[-(i-1) / \alpha]_{m+1}\right),
\end{aligned}
$$

the last equation being a direct consequence of the identity $m[x]_{m-1}=[x+1]_{m}-$ $[x]_{m}$.

For instance we have

$$
d_{1}=\frac{p_{2}^{*}}{2}, \quad d_{2}=\frac{p_{3}^{*}}{3}+\frac{p_{2}^{*}}{2}, \quad d_{3}=\frac{p_{4}^{*}}{4}+p_{3}^{*}+\frac{p_{2}^{*}}{2} .
$$

For each element $f \in \mathbf{S}^{*}[X]$ we define its so called leading symmetric term $[f] \in \mathbf{S}[X]$ as the highest degree homogeneous part of $f$. For instance we have $\left[p_{k}^{\star}\right]=p_{k}$. The map $f \rightarrow[f]$ provides an isomorphism of the graded algebra associated to the filtered algebra $\mathbf{S}^{*}[X]$ onto the symmetric algebra $\mathbf{S}[X]$. Assuming that the leading terms $\left[f_{1}\right],\left[f_{2}\right], \ldots,\left[f_{n}\right]$ of a sequence $f_{1}, f_{2}, \ldots, f_{n}$ generate $\mathbf{S}[X]$, this sequence itself generates $\mathbf{S}^{*}[X]$.

Now for $k \geq 1$ as a consequence of Lemma 7.1,

$$
\left[d_{k}\right]=\frac{\left[p_{k+1}^{*}\right]}{k+1}=\frac{p_{k+1}}{k+1} .
$$

Thus the shifted symmetric functions $p_{1}^{*}=p_{1}$ and $d_{k}, k \geq 1$, algebraically generate $\mathbf{S}^{*}[X]$. In other words any $f \in \mathbf{S}^{*}[\mathcal{P}]$ may be expanded in terms of the functions $|\lambda|^{r} d_{\mu}(\lambda)$, with $r \geq 0$ and $\mu$ any partition.

But as seen before, $d_{\mu}(\lambda)=p_{\mu}\left(A_{\lambda}\right)$ and there is no reason that we shall restrict to the basis of power sums in the alphabet $A_{\lambda}$. Any other basis of the symmetric algebra may be convenient. Therefore any $f \in \mathbf{S}^{*}[\mathcal{P}]$ may be expanded in terms of the functions $|\lambda|^{r} b_{\mu}\left(A_{\lambda}\right)$, with $r \geq 0$ and $b_{\mu}$ any linear basis of the symmetric algebra $\mathbf{S}$, viewed abstractly.

Finally we have proved

Theorem 7.2. Let $G=\mathbf{F}[w]$ be the field of polynomials in some indeterminate $w$ with coefficients in $\mathbf{F}$. The evaluation map $p \otimes f \rightarrow p(|\lambda|) f\left(A_{\lambda}\right)$ is an isomorphism of $G \otimes \mathbf{S}$ onto $\mathbf{S}^{*}[\mathcal{P}]$.

Let us give a simple but enlightening example. Recall that the generalized raising and lowering factorials are given by

$$
(x)_{\lambda}=\prod_{a \in A_{\lambda}}(x+a), \quad[x]_{\lambda}=\prod_{a \in A_{\lambda}}(x-a) .
$$


Then let us define ([14], p. 64)

$$
(x)_{\lambda}=\sum_{k \geq 0} c_{k}(\lambda) x^{|\lambda|-k}, \quad \frac{1}{[x]_{\lambda}}=\sum_{k \geq 0} C_{k}(\lambda) \frac{1}{x^{|\lambda|+k}} .
$$

The quantities $c_{k}(\lambda)$ and $C_{k}(\lambda)$ are generalizations of Stirling numbers of the first and second kind, since when $\lambda$ is a row-partition $(n)$, they are $|s(n, k)|$ and $S(n+k-1, n-1)$, respectively.

We know immediately that $c_{k}(\lambda)$ and $C_{k}(\lambda)$ are shifted symmetric functions, i.e. belong to $\mathbf{S}^{*}[\mathcal{P}]$, and that they satisfy

$$
c_{k}(\lambda)=\sum_{|\mu|=k}(-1)^{k-l(\mu)} \frac{1}{z_{\mu}} d_{\mu}(\lambda), \quad C_{k}(\lambda)=\sum_{|\mu|=k} \frac{1}{z_{\mu}} d_{\mu}(\lambda) .
$$

Indeed their definitions are merely the generating functions for elementary and complete symmetric functions of the alphabet $A_{\lambda}$ since

$$
\begin{gathered}
(x)_{\lambda}=\prod_{a \in A_{\lambda}}(x+a)=x^{|\lambda|} E_{1 / x}\left(A_{\lambda}\right)=\sum_{k \geq 0} x^{|\lambda|-k} e_{k}\left(A_{\lambda}\right), \\
\frac{1}{[x]_{\lambda}}=\prod_{a \in A_{\lambda}} \frac{1}{x-a}=\frac{1}{x^{|\lambda|}} H_{1 / x}\left(A_{\lambda}\right)=\sum_{k \geq 0} \frac{1}{x^{|\lambda|+k}} h_{k}\left(A_{\lambda}\right) .
\end{gathered}
$$

Thus it is enough to apply the classical Cauchy formulas ([19], p. 25) to get

$$
\begin{aligned}
c_{k}(\lambda) & =e_{k}\left(A_{\lambda}\right)=\sum_{|\mu|=k}(-1)^{k-l(\mu)} \frac{1}{z_{\mu}} p_{\mu}\left(A_{\lambda}\right), \\
C_{k}(\lambda) & =h_{k}\left(A_{\lambda}\right)=\sum_{|\mu|=k} \frac{1}{z_{\mu}} p_{\mu}\left(A_{\lambda}\right) .
\end{aligned}
$$

\section{Application to Jack polynomials}

The reference for Jack polynomials is Chapter 6, Section 10 of the book by Macdonald [19]. Let $\alpha$ be some fixed positive real number. The Jack polynomials $J_{\lambda}^{\alpha}$ form a basis of $\mathbf{F} \otimes \mathbf{S}$, the algebra of symmetric functions with coefficients in $\mathbf{F}$.

Jack polynomials satisfy the following generalization of the Pieri formula [25]. For any partition $\lambda$ and any integer $i$ such that $1 \leq i \leq l(\lambda)+1$, denote $\lambda^{(i)}$ the partition $\mu$, if it exists, such that $\mu_{j}=\lambda_{j}$ for $j \neq i$ and $\mu_{i}=\lambda_{i}+1$. Then we have

$$
e_{1} J_{\lambda}^{\alpha}=\sum_{i=1}^{l(\lambda)+1} c_{i}^{\alpha}(\lambda) J_{\lambda^{(i)}}^{\alpha} .
$$

The Pieri coefficients $c_{i}^{\alpha}(\lambda)$ have the following analytic expression [18]:

$$
c_{i}^{\alpha}(\lambda)=\frac{1}{\alpha \lambda_{i}+l(\lambda)-i+2} \prod_{\substack{j=1 \\ j \neq i}}^{l(\lambda)+1} \frac{\alpha\left(\lambda_{i}-\lambda_{j}\right)+j-i+1}{\alpha\left(\lambda_{i}-\lambda_{j}\right)+j-i} .
$$

For any integer $r \geq 0$ we define

$$
s_{r}(\lambda)=\sum_{i=1}^{l(\lambda)+1}\left(\lambda_{i}-\frac{i-1}{\alpha}\right)^{r} c_{i}^{\alpha}(\lambda) .
$$


In [14 we gave the values of $s_{r}(\lambda)$ up to $r=9$. For instance one has

$$
s_{0}(\lambda)=1, \quad s_{1}(\lambda)=0, \quad s_{2}(\lambda)=|\lambda| / \alpha, \quad s_{3}(\lambda)=2 d_{1}(\lambda) / \alpha+|\lambda|(\alpha-1) / \alpha^{2} .
$$

Since $c_{i}^{\alpha}(\lambda)$ is a rational function of the variables $\left\{\lambda_{i}-i / \alpha\right\}$, it is not obvious that $s_{r}(\lambda)$ is a shifted symmetric function, i.e. a polynomial in $\left\{\lambda_{i}-i / \alpha\right\}$. A proof has been given by Macdonald ([14], Theorem 6.1, p. 69). A more direct proof is given below, together with a new explicit formula.

In [14 (Conjecture 6.2, p. 70) we stated the following conjecture.

Conjecture. For any partition $\lambda$ and any integer $r \geq 0$ one has

$$
s_{r}(\lambda)=\sum_{i=0}^{[r / 2]} \sum_{j=0}^{r-2 i} \sum_{k=0}^{\min (i, j)} \frac{1}{\alpha^{i}}\left(1-\frac{1}{\alpha}\right)^{r-2 i-j}\left(\begin{array}{c}
|\lambda|+i-1 \\
i-k
\end{array}\right) \sum_{|\rho|=j} u_{i j k}^{\rho}(r) \frac{d_{\rho}(\lambda)}{z_{\rho}}
$$

where the coefficients $u_{i j k}^{\rho}(r)$ are positive integers.

Theorem 8.1. The previous conjecture is true, with

$$
u_{i j k}^{\rho}(r)=\sum_{s=0}^{j}\left\langle\begin{array}{l}
\rho \\
s
\end{array}\right\rangle_{k}\left(\begin{array}{c}
r+s-i-j-1 \\
r-2 i-j
\end{array}\right) .
$$

In other words we have

$$
\begin{aligned}
s_{r}(\lambda)=\sum_{\substack{n, p, q \geq 0 \\
2 n+p+q \leq r}} \frac{1}{\alpha^{n}}\left(1-\frac{1}{\alpha}\right)^{p}\left(\begin{array}{c}
n+p+q-1 \\
p
\end{array}\right) & \\
& \times\left(\sum_{k=0}^{\min (n, r-2 n-p)}\left(\begin{array}{c}
|\lambda|+n-1 \\
n-k
\end{array}\right) F_{r-2 n-p, q, k}(\lambda)\right) .
\end{aligned}
$$

Proof. We shall prove that the quantities $s_{r}(\lambda)$ have the following generating function:

$$
\sum_{r \geq 0} s_{r}(\lambda)\left(\frac{-1}{x}\right)^{r}=\frac{(x-1 / \alpha+1)_{\lambda}}{(x-1 / \alpha)_{\lambda}} \frac{(x)_{\lambda}}{(x+1)_{\lambda}} .
$$

Then the statement will immediately follow from Corollary 5.2 with $y=-1 / \alpha$. To prove this generating function we apply Lagrange Lemma for the following two alphabets:

$$
\begin{gathered}
A=\left\{a_{i}=\alpha \lambda_{i}-i+1, i=1, \ldots, l(\lambda)+1\right\}, \\
B=\left\{b_{i}=\alpha \lambda_{i}-i, i=1, \ldots, l(\lambda)\right\} .
\end{gathered}
$$

Then it is obvious that

$$
c_{i}^{\alpha}(\lambda)=\frac{\prod_{b \in B}\left(a_{i}-b\right)}{\prod_{c \in A, c \neq a_{i}}\left(a_{i}-c\right)},
$$

and the Lagrange Lemma yields

$$
\alpha^{r} s_{r}(\lambda)=h_{r}(A-B) .
$$


The generating function of $\alpha^{r} s_{r}(\lambda)$ is thus

$$
\begin{aligned}
H_{z}(A-B) & =\frac{\prod_{b \in B}(1-z b)}{\prod_{a \in A}(1-z a)} \\
& =\prod_{i=1}^{l(\lambda)} \frac{1-z\left(\alpha \lambda_{i}-i\right)}{1-z\left(\alpha \lambda_{i}-i+1\right)} \frac{1}{1+z l(\lambda)} .
\end{aligned}
$$

With $\alpha z=-1 / x$ we obtain

$$
\sum_{r \geq 0} s_{r}(\lambda)\left(\frac{-1}{x}\right)^{r}=\prod_{i=1}^{l(\lambda)} \frac{x+\lambda_{i}-i / \alpha}{x+\lambda_{i}-(i-1) / \alpha} \frac{x}{x-l(\lambda) / \alpha} .
$$

Since

$$
\frac{(u+1)_{\lambda}}{(u)_{\lambda}}=\prod_{i=1}^{l(\lambda)} \prod_{j=1}^{\lambda_{i}} \frac{u+j-(i-1) / \alpha}{u+j-1-(i-1) / \alpha}=\prod_{i=1}^{l(\lambda)} \frac{u+\lambda_{i}-(i-1) / \alpha}{u-(i-1) / \alpha},
$$

we conclude easily.

Remark 8.2. As already emphasized, Theorem 8.1 gives the expansion of the shifted symmetric function $s_{r}(\lambda)$ over the basis $|\lambda|^{r} p_{\mu}\left(A_{\lambda}\right)$. An open problem is to find explicit expansions over a different basis of the symmetric algebra $\mathbf{S}\left[A_{\lambda}\right]$, for instance $|\lambda|^{r} m_{\mu}\left(A_{\lambda}\right)$.

\section{Application to Shifted JaCK polynomials}

For any partition $\mu$ there exists a shifted symmetric function $\widetilde{J_{\mu}^{\alpha}}$ such that

(i) degree $\widetilde{J_{\mu}^{\alpha}}=|\mu|$,

(ii) $\widetilde{J_{\mu}^{\alpha}}(\lambda)=0$ except if $\mu_{i} \leq \lambda_{i}$ for any $i$, and $\widetilde{J_{\mu}^{\alpha}}(\mu) \neq 0$.

This function is called the shifted Jack polynomial associated with $\mu$ [7, 8, 21, 22, 23, 24]. It is unique up to the value of $\widetilde{J_{\mu}^{\alpha}}(\mu)$. When conveniently normalized, $\widetilde{J_{\mu}^{\alpha}}(\lambda)$ is merely the generalized binomial coefficient

$$
\frac{\widetilde{J_{\mu}^{\alpha}}(\lambda)}{\widetilde{J_{\mu}^{\alpha}}(\mu)}=\left(\begin{array}{l}
\lambda \\
\mu
\end{array}\right)_{\alpha}
$$

appearing in the generalized binomial formula for Jack polynomials [3, 16, 17, 22].

We mention the following two remarkable facts:

(a) In the definition of $\widetilde{J_{\mu}^{\alpha}}$, the overdetermined system of linear conditions (ii) may be replaced by the weaker conditions

(iii) $\widetilde{J_{\mu}^{\alpha}}(\lambda)=0$ except if $|\mu| \leq|\lambda|$, and $\widetilde{J_{\mu}^{\alpha}}(\mu) \neq 0$.

(b) For some constant $h_{\mu}$ one has $\left[\widetilde{J_{\mu}^{\alpha}}\right]=h_{\mu} J_{\mu}^{\alpha}$.

For any partition $\lambda$ and any integer $i$ such that $1 \leq i \leq l(\lambda)$, we denote $\lambda_{(i)}$ the partition $\mu$, if it exists, such that $\mu_{j}=\lambda_{j}$ for $j \neq i$ and $\mu_{i}=\lambda_{i}-1$. In [16], p. 320 (see also [17], Theorem 5), we have proved that

$$
\left(\begin{array}{c}
\lambda \\
\lambda_{(i)}
\end{array}\right)_{\alpha}=\left(\lambda_{i}+\frac{l(\lambda)-i}{\alpha}\right) \prod_{\substack{j=1 \\
j \neq i}}^{l(\lambda)} \frac{\alpha\left(\lambda_{i}-\lambda_{j}\right)+j-i-1}{\alpha\left(\lambda_{i}-\lambda_{j}\right)+j-i} .
$$


For any integer $r \geq 0$ we introduce the shifted symmetric function

$$
\sigma_{r}(\lambda)=\sum_{i=1}^{l(\lambda)}\left(\lambda_{i}-\frac{i-1}{\alpha}\right)^{r}\left(\begin{array}{c}
\lambda \\
\lambda_{(i)}
\end{array}\right)_{\alpha} .
$$

As shown in [16], Theorem 8 (see also [17], Theorem 3) we have $\sigma_{0}(\lambda)=|\lambda|$. But even the computation of the first values

$$
\sigma_{1}(\lambda)=2 d_{1}(\lambda)+|\lambda|, \quad \sigma_{2}(\lambda)=3 d_{2}(\lambda)+\left(3+\frac{1}{\alpha}\right) d_{1}(\lambda)+|\lambda|-\frac{1}{\alpha}\left(\begin{array}{c}
|\lambda| \\
2
\end{array}\right)
$$

seems rather difficult, except by the method given below.

Theorem 9.1. We have

$$
\sigma_{r}(\lambda)=c_{r+1}-\alpha c_{r+2},
$$

with

$$
\begin{aligned}
c_{r}=\sum_{\substack{n, p, q \geq 0 \\
2 n+p+q \leq r}}\left(-\frac{1}{\alpha}\right)^{n}(1+ & \left.\frac{1}{\alpha}\right)^{p}\left(\begin{array}{c}
n+p+q-1 \\
p
\end{array}\right) \\
& \times\left(\sum_{k=0}^{\min (n, r-2 n-p)}\left(\begin{array}{c}
|\lambda|+n-1 \\
n-k
\end{array}\right) F_{r-2 n-p, q, k}(\lambda)\right) .
\end{aligned}
$$

Proof. We shall prove that the quantities $\sigma_{r}(\lambda)$ have the following generating function:

$$
\sum_{r \geq 0} \sigma_{r}(\lambda)\left(\frac{-1}{x}\right)^{r}=-x(\alpha x+1)\left(\frac{(x+1 / \alpha+1)_{\lambda}}{(x+1 / \alpha)_{\lambda}} \frac{(x)_{\lambda}}{(x+1)_{\lambda}}-1\right) .
$$

Then the statement will immediately follow from Corollary 5.2 with $y=1 / \alpha$. To prove this generating function we apply Lagrange Lemma for the following two alphabets:

$$
\begin{aligned}
& A=\left\{a_{i}=\alpha \lambda_{i}-i+1, i=1, \ldots, l(\lambda)\right\}, \\
& B=\left\{b_{i}=\alpha \lambda_{i}-i+2, i=1, \ldots, l(\lambda)+1\right\} .
\end{aligned}
$$

Then it is obvious that

$$
\left(\begin{array}{c}
\lambda \\
\lambda_{(i)}
\end{array}\right)_{\alpha}=-\frac{1}{\alpha} \frac{\prod_{b \in B}\left(a_{i}-b\right)}{\prod_{c \in A, c \neq a_{i}}\left(a_{i}-c\right)},
$$

and the Lagrange Lemma yields

$$
-\alpha^{r+1} \sigma_{r}(\lambda)=h_{r+2}(A-B) .
$$

The generating function of $-\alpha^{r+1} \sigma_{r}(\lambda)$ is thus

$$
-\sum_{r \geq 0} \alpha^{r+1} z^{r} \sigma_{r}(\lambda)=\frac{1}{z^{2}} \sum_{r \geq 2} z^{r} h_{r}(A-B)=\frac{1}{z^{2}}\left(H_{z}(A-B)-z h_{1}(A-B)-1\right) .
$$

But we have

$$
\begin{aligned}
H_{z}(A-B) & =\frac{\prod_{b \in B}(1-z b)}{\prod_{a \in A}(1-z a)} \\
& =\prod_{i=1}^{l(\lambda)} \frac{1-z\left(\alpha \lambda_{i}-i+2\right)}{1-z\left(\alpha \lambda_{i}-i+1\right)}(1+z(l(\lambda)-1)),
\end{aligned}
$$


and it is easily checked that

$$
h_{1}(A-B)=\sum_{i=1}^{l(\lambda)}\left(a_{i}-b_{i}\right)+l(\lambda)-1=-1 .
$$

With $\alpha z=-1 / x$ we obtain

$$
\sum_{r \geq 0} \sigma_{r}(\lambda)\left(\frac{-1}{x}\right)^{r}=-\alpha x^{2}\left(\prod_{i=1}^{l(\lambda)} \frac{x+\lambda_{i}-(i-2) / \alpha}{x+\lambda_{i}-(i-1) / \alpha} \frac{x-(l(\lambda)-1) / \alpha}{x}-\frac{1}{\alpha x}-1\right) .
$$

The right-hand side can be written as

$$
-\alpha x(x-(l(\lambda)-1) / \alpha) \frac{(x+1 / \alpha+1)_{\lambda}}{(x+1 / \alpha)_{\lambda}} \frac{(x)_{\lambda}}{(x+1)_{\lambda}} \prod_{i=1}^{l(\lambda)} \frac{x-(i-2) / \alpha}{x-(i-1) / \alpha}+x+\alpha x^{2} .
$$

Hence the result.

\section{Probability distributions}

Let us briefly outline the connections of these results with probability distributions on the set of Young diagrams [4, 5, 20.

We denote by $\mathcal{P}_{n}$ the set of Young (i.e. Ferrers) diagrams with $n$ cells, and by $\mathcal{P}=\bigcup \mathcal{P}_{n}$ the lattice of all Young diagrams ordered by inclusion. We write $\lambda \nearrow \Lambda$ if the diagram $\Lambda$ is obtained from $\lambda$ by adding a cell, i.e. $\Lambda=\lambda^{(i)}$ for some row $i$. We regard such a pair $(\lambda, \Lambda)$ as an "oriented edge" of the "infinite graph" $\mathcal{P}$.

We use the Pieri formula

$$
e_{1} J_{\lambda}^{\alpha}=\sum_{\Lambda: \lambda \nearrow \Lambda} \kappa(\lambda, \Lambda) J_{\Lambda}^{\alpha}
$$

to define a "multiplicity function" $\kappa$ on the set of edges of $\mathcal{P}$. A function $\Phi$ defined on $\mathcal{P}$ is said to be harmonic if for all vertices $\lambda \in \mathcal{P}$ one has

$$
\Phi(\lambda)=\sum_{\Lambda: \lambda \nearrow \Lambda} \kappa(\lambda, \Lambda) \Phi(\Lambda)
$$

Let $\varphi$ be the particular case corresponding to the initial condition $\varphi(\emptyset)=1$. Then (4], Lemma 7.2) one has $\varphi(\lambda)=1$ for any $\lambda \in \mathcal{P}$.

Similarly we define a "dimension function" $\operatorname{dim} \Lambda$ by the following recurrence:

$$
\operatorname{dim} \Lambda=\sum_{\lambda: \lambda \nearrow \Lambda} \kappa(\lambda, \Lambda) \operatorname{dim} \lambda
$$

with the initial condition $\operatorname{dim} \emptyset=1$. Then ([4], Corollary 6.10 ) one has

$$
\operatorname{dim} \lambda=|\lambda| ! \frac{\alpha^{|\lambda|}}{j_{\lambda}^{\alpha}}
$$

with $j_{\lambda}^{\alpha}=\left\langle J_{\lambda}^{\alpha}, J_{\lambda}^{\alpha}\right\rangle$ the norm of $J_{\lambda}^{\alpha}([25]$, p. 97$)$.

It is shown in [4, 5] that for any oriented edge $\lambda \nearrow \Lambda$ the quantities

$$
p_{\lambda}(\Lambda)=\kappa(\lambda, \Lambda) \frac{\varphi(\Lambda)}{\varphi(\lambda)}, \quad q_{\Lambda}(\lambda)=\kappa(\lambda, \Lambda) \frac{\operatorname{dim} \lambda}{\operatorname{dim} \Lambda}
$$

define two discrete "transition" $p_{\lambda}$ and "co-transition" $q_{\Lambda}$ probability distributions, associated with the Young diagrams $\lambda$ and $\Lambda$, respectively. 
But as a consequence of [17], Theorem 2 for any oriented edge $\lambda \nearrow \Lambda$ one has

$$
\kappa(\lambda, \Lambda)=\alpha \frac{j_{\lambda}^{\alpha}}{j_{\Lambda}^{\alpha}}\left(\begin{array}{l}
\Lambda \\
\lambda
\end{array}\right)_{\alpha},
$$

so that with our previous notations, we can write

$$
p_{\lambda}\left(\lambda^{(i)}\right)=c_{i}^{\alpha}(\lambda), \quad q_{\Lambda}\left(\Lambda_{(i)}\right)=\frac{1}{|\Lambda|}\left(\begin{array}{c}
\Lambda \\
\Lambda_{(i)}
\end{array}\right)_{\alpha} .
$$

Now let us consider the $\alpha$-content random variable $c$ defined on any cell $(i, j)$ by $c(i, j)=j-1-(i-1) / \alpha$. If $\lambda / \Lambda$ is an oriented edge (i.e. $\Lambda=\lambda^{(i)}$ for some row i) we have

$$
c(\Lambda \backslash \lambda)=\lambda_{i}-(i-1) / \alpha=\Lambda_{i}-1-(i-1) / \alpha .
$$

Hence the moments of the random variable $c$ with respect to the transition and co-transition distributions are respectively

$$
\begin{aligned}
\sum_{\Lambda: \lambda \nearrow \Lambda} c(\Lambda \backslash \lambda)^{r} p_{\lambda}(\Lambda) & =s_{r}(\lambda), \\
\sum_{\lambda: \lambda / \Lambda} c(\Lambda \backslash \lambda)^{r} q_{\Lambda}(\lambda) & =\frac{1}{|\Lambda|} \sum_{k=0}^{r}(-1)^{r-k}\left(\begin{array}{l}
r \\
k
\end{array}\right) \sigma_{k}(\Lambda) .
\end{aligned}
$$

Our results in Sections 8 and 9 thus amount to give an explicit evaluation of these moments.

If $\alpha=1$ the multiplicities are given by $\kappa(\lambda, \Lambda)=H_{\lambda} / H_{\Lambda}$, with $H_{\lambda}$ the classical hook polynomial $\prod_{i, j \in \lambda}\left(\lambda_{i}+\lambda_{j}^{\prime}-i-j+1\right)$, and $\lambda^{\prime}$ the partition conjugate to $\lambda$. Let $f_{\lambda}=|\lambda| ! / H_{\lambda}$ denote the dimension of the irreducible representation of the symmetric group associated to $\lambda$ (i.e. the number of standard tableaux of shape $\lambda)$. Then one recovers the classical distributions

$$
p_{\lambda}(\Lambda)=\frac{1}{|\Lambda|} \frac{f_{\Lambda}}{f_{\lambda}}, \quad q_{\Lambda}(\lambda)=\frac{f_{\lambda}}{f_{\Lambda}} .
$$

Our results are new even in this classical case.

In [4] it was shown that Young diagrams must be interpreted as a special case of "pairs of interlacing sequences", and that transition and co-transition distributions can be defined in this more general frame. It is likely that our explicit evaluations can be easily translated in this context.

\section{Rows AND COLUMnS}

To conclude we give the proof of two conjectures stated in Sections 9 and 10 of 15] (all conjectures of these sections will then be established). This result was not included in [10], though it is a direct consequence of relation (5.1) proved there. We shall need the following lemma.

Lemma 11.1. With $s(n, k)$ the Stirling numbers of the first kind, we have

$$
\frac{1}{x^{k}}=\sum_{n \geq k} s(n-1, k-1) \frac{1}{[x]_{n}} .
$$


Proof. Let $\Delta$ be the finite difference operator defined by $\Delta f(y)=f(y+1)-f(y)$. We write the Newton interpolation formula

$$
f(y)=\sum_{n \geq 0}[y]_{n} \frac{\Delta^{n} f(0)}{n !},
$$

for $f(y)=x /(x-y)=\sum_{r \geq 0} y^{r} / x^{r}$. This yields immediately

$$
f(y)=\sum_{n \geq 0}[y]_{n} \frac{x}{[x]_{n+1}} .
$$

The result follows by identifying coefficients of $y^{k}$.

Theorem 11.2. For any integer $p \geq 0$ we have

$$
\begin{gathered}
\left(\begin{array}{c}
\lambda \\
(p)
\end{array}\right)_{\alpha}=\frac{1}{(1 / \alpha)_{p}} \sum_{0<i+j \leq p} \frac{1}{\alpha^{i}} s(p-1, i+j-1)\left(\sum_{k=0}^{\min (i, j)}\left(\begin{array}{c}
|\lambda|-j \\
i-k
\end{array}\right) F_{j k}(\lambda)\right), \\
\left(\begin{array}{c}
\lambda \\
1^{p}
\end{array}\right)_{\alpha}=\frac{1}{(\alpha)_{p}} \sum_{0<i+j \leq p}(-1)^{j} \alpha^{i+j} s(p-1, i+j-1)\left(\sum_{k=0}^{\min (i, j)}\left(\begin{array}{c}
|\lambda|-j \\
i-k
\end{array}\right) F_{j k}(\lambda)\right) .
\end{gathered}
$$

Proof. By the property of duality ([16], p. 320)

$$
\left(\begin{array}{c}
\lambda \\
(p)
\end{array}\right)_{\alpha}=\left(\begin{array}{c}
\lambda^{\prime} \\
1^{p}
\end{array}\right)_{1 / \alpha}
$$

and using $d_{k}\left(\lambda^{\prime} ; 1 / \alpha\right)=(-\alpha)^{k} d_{k}(\lambda ; \alpha)$, hence $F_{j k}\left(\lambda^{\prime} ; 1 / \alpha\right)=(-\alpha)^{j} F_{j k}(\lambda ; \alpha)$, it is enough to prove the second formula. The generalized Chu-Vandermonde formula proved in [15] (Theorem 12.1, p. 161) reads

$$
\frac{(y+1)_{\lambda}}{(y)_{\lambda}}=\sum_{\mu}(-1)^{\mu}\left(\begin{array}{l}
\lambda \\
\mu
\end{array}\right)_{\alpha} \frac{(-1)_{\mu}}{(y)_{\mu}}=\sum_{p \geq 0}\left(\begin{array}{c}
\lambda \\
1^{p}
\end{array}\right)_{\alpha} \frac{(\alpha)_{p}}{[\alpha y]_{p}},
$$

since $(-1)_{\mu}=0$ if $\mu_{1}>1$. On the other hand relation (5.1) implies

$$
\frac{(y+1)_{\lambda}}{(y)_{\lambda}}=\sum_{i, j \geq 0}(-1)^{j} \frac{1}{y^{i+j}}\left(\sum_{k=0}^{\min (i, j)}\left(\begin{array}{c}
|\lambda|-j \\
i-k
\end{array}\right) F_{j k}(\lambda)\right) .
$$

Using Lemma 11.1, we conclude by comparison.

\section{ACKNOWLEDGEMENTS}

It is a pleasure to thank Alain Lascoux for pointing out the relevance of Lagrange interpolation.

\section{REFERENCES}

[1] F. Jouhet, Jiang Zeng, Généralisation de formules de type Waring, Sém. Lothar. Combin. 44 (2000), Article B44g. MR 2002b:05134

[2] F. Jouhet, B. Lass, Jiang Zeng, Sur une généralisation des coefficients binomiaux, http:// arXiv.org/abs/math.CO/0303025.

[3] J. Kaneko, Selberg integrals and hypergeometric functions associated with Jack polynomials, SIAM J. Math. Anal. 24 (1993), 1086-1110. MR 94h:33010

[4] S. Kerov, Anisotropic Young diagrams and Jack symmetric functions, Funct. Anal. Appl. 34 (2000), http://arXiv.org/abs/math.CO/9712267 MR 2001f:05158 
[5] S. Kerov, The boundary of Young lattice and random Young tableaux, DIMACS Ser. Discrete Math. Theoret. Comput. Sci. 24 (1996), 133-158. MR 96i:05177

[6] S. Kerov, A. Okounkov, G. Olshanski, The boundary of Young graph with Jack edge multiplicities, Int. Math. Res. Not. 1998 (4), 173-199. MR 99f:05120

[7] F. Knop, Symmetric and nonsymmetric quantum Capelli polynomials, Comment. Math. Helv. 72 (1997), 84-100. MR 98m:05204

[8] F. Knop, S. Sahi, Difference equations and symmetric polynomials defined by their zeros, Int. Math. Res. Not. 1996 (10), 473-486. MR 99d:05086

[9] A. Lascoux, Notes on interpolation in one and several variables, http://phalanstere.univ$\mathrm{mlv} . \mathrm{fr} / \sim \mathrm{al} /$.

[10] A. Lascoux, M. Lassalle, Une identité remarquable en théorie des partitions, Math. Ann. 318 (2000), 299-313. MR 2001k:05204

[11] M. Lassalle, A new family of positive integers, Ann. Comb. 6 (2002), 399-405.

[12] M. Lassalle, Une identité en théorie des partitions, J. Combin. Theory, Ser. A, 89 (2000), 270-288. MR 2001a:05013

[13] M. Lassalle, Quelques conjectures combinatoires relatives à la formule classique de ChuVandermonde, Adv. in Appl. Math. 21 (1998), 457-472. MR 99i:05019

[14] M. Lassalle, Some combinatorial conjectures for Jack polynomials, Ann. Comb. 2 (1998), 61-83. MR 2000m:05230

[15] M. Lassalle, Some combinatorial conjectures for shifted Jack polynomials, Ann. Comb. 2 (1998), 145-163. MR 2000m:05231

[16] M. Lassalle, Coefficients binomiaux généralisés et polynômes de Macdonald, J. Funct. Anal. 158 (1998), 289-324. MR 2000a:33028

[17] M. Lassalle, Une formule du binôme généralisée pour les polynômes de Jack, C. R. Acad. Sci. Paris, Sér. I Math. 310 (1990), 253-256. MR 91c:05193

[18] M. Lassalle, Une formule de Pieri pour les polynômes de Jack, C. R. Acad. Sci. Paris, Sér. I Math. 309 (1989), 941-944. MR 91c:05192

[19] I.G. Macdonald, Symmetric functions and Hall polynomials, Clarendon Press, second edition, Oxford, 1995. MR 96h:05207

[20] G. Olshanski, A. Regev, Random Young tableaux and combinatorial identities, http:// arXiv.org/abs/math.CO/0106074.

[21] A. Okounkov, (Shifted) Macdonald polynomials, q-integral representation and combinatorial formula, Compositio. Math. 112 (1998), 147-182. MR 99h:05120

[22] A. Okounkov, G. Olshanski, Shifted Jack polynomials, binomial formula and applications, Math. Res. Lett. 4 (1997), 69-78. MR 98h:05177

[23] A. Okounkov, G. Olshanski, Shifted Schur functions, St. Petersburg Math. J. 9 (1998), 239300. MR 99f:05118

[24] S. Sahi, Interpolation, integrality and a generalization of Macdonald's polynomials, Int. Math. Res. Not. 1996 (10), 457-471. MR 99j:05189b

[25] R. P. Stanley, Some combinatorial properties of Jack symmetric functions, Adv. Math. $\mathbf{7 7}$ (1989), 76-115. MR 90g:05020

[26] S. Veigneau, ACE, an Algebraic Combinatorics Environment for the computer algebra system Maple, http://phalanstere.univ-mlv.fr/ ace/.

[27] Jiang Zeng, private communication.

Centre National de la Recherche Scientifique, Institut Gaspard Monge, Université de Marne-la-Vallée, 77454 Marne-la-Vallée Cedex, France

E-mail address: lassalle@univ-mlv.fr

URL: http://igm.univ-mlv.fr/〜 lassalle/index.html 